\title{
Renoprotective Benefit of Tolvaptan in Acute Decompensated Heart Failure Patients With Loop Diuretic- Resistant Status
}

\author{
Tomohiko Yamamoto $^{\mathrm{a}, \mathrm{c}}$, Shin-ichiro Miura ${ }^{\mathrm{b}}$, Kazuyuki Shirai ${ }^{\mathrm{a}}$, \\ Hidenori Urata ${ }^{\mathrm{a}}$
}

\begin{abstract}
Background: While reduction of accumulated body fluid using loop diuretics is a commonly used therapeutic option for acute heart failure (AHF), some patients, especially those with chronic kidney disease (CKD), show significantly poor treatment response to loop diuretics. Tolvaptan (TLV) has shown effectiveness against AHF in several studies. We have been using TLV for AHF treatment, and it displayed favorable outcome even in patients with CKD. This study aimed to assess the therapeutic effectiveness of TLV in AHF patients.
\end{abstract}

Methods: Ninety-nine AHF patients who were hospitalized were assessed retrospectively. Patients were divided into two groups: TLV treatment (TLV group, $\mathrm{n}=39$ ) and conventional treatment (non-TLV group, $n=60$ ). We retrospectively examined the efficacy of TLV combination therapy for renal insufficiency complications and loop diuretic-resistant AHF patients, and the detail analysis was performed for heart failure with preserved ejection fraction (HFpEF) or reduced ejection fraction (HFrEF) in patients.

Results: Changes in serum electrolyte levels before and after the treatment were similar in both groups. Although the patients in the TLV group at baseline displayed significantly lower estimated glomerular filtration rate (eGFR) indicating renal insufficiency probably due to higher dose of loop diuretics, the incidence of worsening renal function (WRF) was significantly lower than those in non-TLV group in HFpEF (TLV: $2.5 \%$ vs. non-TLV: $15.4 \%, \mathrm{P}=0.01)$. We performed logistic regression analysis and found that TLV was an independent contributing factor for reducing WRF (odds ratio: 0.14, 95\% $\mathrm{CI}$ : 0.02 - 0.98, $\mathrm{P}=0.04$ ).

Conclusions: Our results suggest that TLV application in acute stage may be renoprotective for AHF patients with $\mathrm{CKD}$, especially in HF$\mathrm{pEF}$.

Manuscript submitted October 22, 2018, accepted November 16, 2018

aDepartment of Cardiovascular Diseases, Fukuoka University Chikushi Hospital, 1-1-1 Zokumyouin, Chikushino, Fukuoka 818-8502, Japan

bepartment of Cardiology, Fukuoka University School of Medicine, 7-45-1 Nanakuma, Jonan-ku, Fukuoka 814-0180, Japan

${ }^{\mathrm{c} C}$ Corresponding Author: Tomohiko Yamamoto, Department of Cardiovascular Diseases, Fukuoka University Chikushi Hospital, 1-1-1 Zokumyouin, Chikushino, Fukuoka 818-8502, Japan. Email: tomohiko0326@gmail.com

doi: https://doi.org/10.14740/jocmr3671
Keywords: Heart failure; HFpEF; Diuretic; Vasopressin; Tolvaptan; Worsening renal function

\section{Introduction}

The number of heart failure (HF) patients is increasing with aging of the Japanese society, and it is expected to affect more than 1.3 million patients by 2030 [1]. Acute heart failure (AHF) is a major cause of hospitalization of elderly patients, and its prognosis remains unfavorable $[2,3]$. The AHF treatment guideline published by The Japanese Circulation Society, recommended loop diuretics and carperitide for the treatment of congestive HF patients. However, we often see patients who have insufficient response to these agents, resulting in difficult management of HF.

Tolvaptan (TLV) is a selective vasopressin V2 receptor antagonist, which competes with antidiuretic hormone vasopressin in the V2 receptor of the kidney collecting duct. This antagonistic action of TLV on the V2 receptor inhibits apoclin-2 activation, resulting in suppression of water resorption at the collecting duct, and increases water excretion. While hyponatremia is often seen in HF patients, which significantly increases cardiac death [4]; TLV only excretes water and effectively corrects hyponatremia [5]. Renal insufficiency induced by congestive HF treatment is called worsening renal function (WRF), which is defined as increase in serum creatinine $(\mathrm{Cr})$ levels at $0.3 \mathrm{mg} /$ $\mathrm{dL}$ or higher. The DOSE trial which compared administration routes between intravenous injection and continuous drip infusion of loop diuretics, and high- and low-dose loop diuretics in patients with acute uncompensated HF, showed that the difference in loop diuretic administration routes and doses were not effective, but induced renal insufficiency. A study suggested the limitation of loop diuretic in AHF treatment [4]. Recent studies showed that reduction of loop diuretic doses in AHF patients can prevent WRF [6-9]. The AQUAMARINE study examined the effectiveness of TLV for AHF patients complicated with renal dysfunction [10], the K-STAR study proved the effectiveness of TLV for patients with renal failure [11], and another study [12] examined the effectiveness of TLV for patients with loop diuretic-resistant HF. These studies suggest that concurrent TLV therapy is a promising treatment option for AHF. TLV combination therapy for renal failure complications and loop diuretic-resistant AHF patients has been proven to be effective, 
but few reports have validated its efficacy with heart failure with preserved ejection fraction $(\mathrm{HFpEF})$ or reduced ejection fraction (HFrEF). We retrospectively examined the efficacy of TLV combination therapy for renal insufficiency complications and loop diuretic-resistant AHF patients, and the detail analysis was performed for HFpEF or HFrEF patients.

\section{Materials and Methods}

\section{Study subjects}

In this single-center, retrospective study, 99 patients with acute decompensated HF, who were hospitalized between April 2015 and December 2016, were recruited and comparatively analyzed. The age of the patient was over 20 years. Patients with acute coronary syndrome and dialysis were excluded. Patients were divided into two groups, 39 patients with concurrent TLV treatment (TLV group) and 60 patients with conventional treatment (non-TLV group). The following endpoints were evaluated: 1) Number of days until improvement of HF (disappearance of clinical congestion (limb edema, lung congestion, and pleural effusion in chest X-ray) and stabilized body weight change for 2 days and more); 2) Total dose of loop diuretics between initiation of treatment and 1 week after the treatment; 3) Changes in blood pressure (systolic blood pressure (SBP); diastolic blood pressure (DBP)) and heart rate (HR) in 1 week; and 4) Changes in renal function (delta blood urea nitrogen $(\triangle \mathrm{BUN})$; delta creatinine $(\Delta \mathrm{Cr})$; delta estimated glomerular filtration rate $(\triangle \mathrm{eGFR})$ ), electrolytes (delta sodium concentration $(\Delta \mathrm{Na})$; delta potassium $(\Delta \mathrm{K}))$, and WRF incidence rate. $\Delta$ indicates the values 1 day after the treatment minus the values at pre-treatment. WRF is defined as $\mathrm{Cr}$ increase of $0.3 \mathrm{mg} / \mathrm{dL}$ or more within 1 week during HF treatment.

The effects of TLV on HFpEF and HFrEF were investigated. The cutoff values of HFpEF and HFrEF were 45\%, which is the average value of left ventricular ejection fraction (LVEF) in ultrasound cardiography.

\section{Statistical analyses}

Statistical analyses were performed using SAS 9.3 (SAS Institute Inc.). Data were shown as the mean \pm standard deviation (SD) or as the median (interquartile range). Categorical and continuous variables were compared between groups by Chisquare analysis and Fisher's exact test and unpaired or paired $t$ test, respectively. Multivariate analysis was performed using a logistic regression analysis for the incidence of WRF. A value of $\mathrm{P}<0.05$ was considered significant.

\section{Results}

\section{Baseline characteristics in the TLV and non-TLV groups}

Table 1 shows the baseline characteristics of the 99 patients
( $\mathrm{n}=39$, TLV group; $\mathrm{n}=60$, non-TLV group). The median age was 80 years in both groups, and more than a half of the patients were men. The mean LVEF was $45 \%$ in both groups. HF severities using New York Heart Association (NYHA) classification revealed that approximately $60 \%$ of the patients were class III or IV, and there was no group difference in patients whose causes of HF were identified. SBP, DBP, and HR at hospitalization were significantly higher in the non-TLV group. In the TLV group, the mean time-lag between initiation of HF treatment and additional TLV administration was 2.7 days.

\section{Medications and biochemical parameters}

There was no difference in concomitant medication at hospitalization in each group. In TLV, the serum $\mathrm{Cr}$ at baseline was higher than that in non-TLV (TLV: $1.3 \mathrm{mg} / \mathrm{dL}$ vs. non-TLV: $0.9 \mathrm{mg} / \mathrm{dL}, \mathrm{P}=0.01$ ) and eGFR was lower (TLV: $35.3 \mathrm{~mL} /$ $\mathrm{min} / 1.73 \mathrm{~m}^{2}$ vs. non-TLV: $\left.48.2 \mathrm{~mL} / \mathrm{min} / 1.73 \mathrm{~m}^{2}, \mathrm{P}=0.003\right)$, indicating that renal function was rather worse in the TLV group. When chronic kidney disease (CKD) is defined as eGFR $<60$ $\mathrm{mL} / \mathrm{min} / 1.73 \mathrm{~m}^{2}, 99 \%$ of TLV and $77 \%$ of non-TLV patients had comorbid CKD. Serum sodium and potassium levels did not show difference between the two groups.

\section{Types of used diuretics and catecholamine}

In concomitant diuretics at the initiation of treatment, carperitide was used in $44 \%$ of the patients in the TLV group. There were no significant differences in the use of other diuretics between the two groups. The usage rate of catecholamine is $18 \%$ in the TLV group and $12 \%$ in the non-TLV group $(\mathrm{P}=0.39)$.

\section{Improvement of HF}

In terms of the number of days until improvement of HF from initiation of the treatment, although the days of improvement in the TLV group appeared to be shorter than that in the nonTLV group, there was no significant difference (Fig. 1a). In terms of average body weight change (Fig. 1b) and cardiac thoracic ratio (CTR) change in chest X-ray (Fig. 1c) during the treatment, there was no significant differences in the two groups except for the CTR reduction in LVEF < 45\% (TLV: $-1.6 \%$, non-TLV: $-4.8 \%, \mathrm{P}=0.001)$.

Total loop diuretic use before hospitalization and 1 week from the start of treatment

Figure 2a shows the average dose of loop diuretics before hospitalization, which shows significant difference in LVEF < 45\% (TLV: $37.9 \mathrm{mg}$, non-TLV: $8.4 \mathrm{mg}, \mathrm{P}<0.001$ ). Figure $2 \mathrm{~b}$ shows the total dose of loop diuretics from treatment initiation to 1 week after the treatment. Although the total dose of loop diuretics in the TLV group was higher than that in the non-TLV group, there was no statistical significant difference. 
Table 1. Patients Characteristics in the TLV and Non-TLV Groups

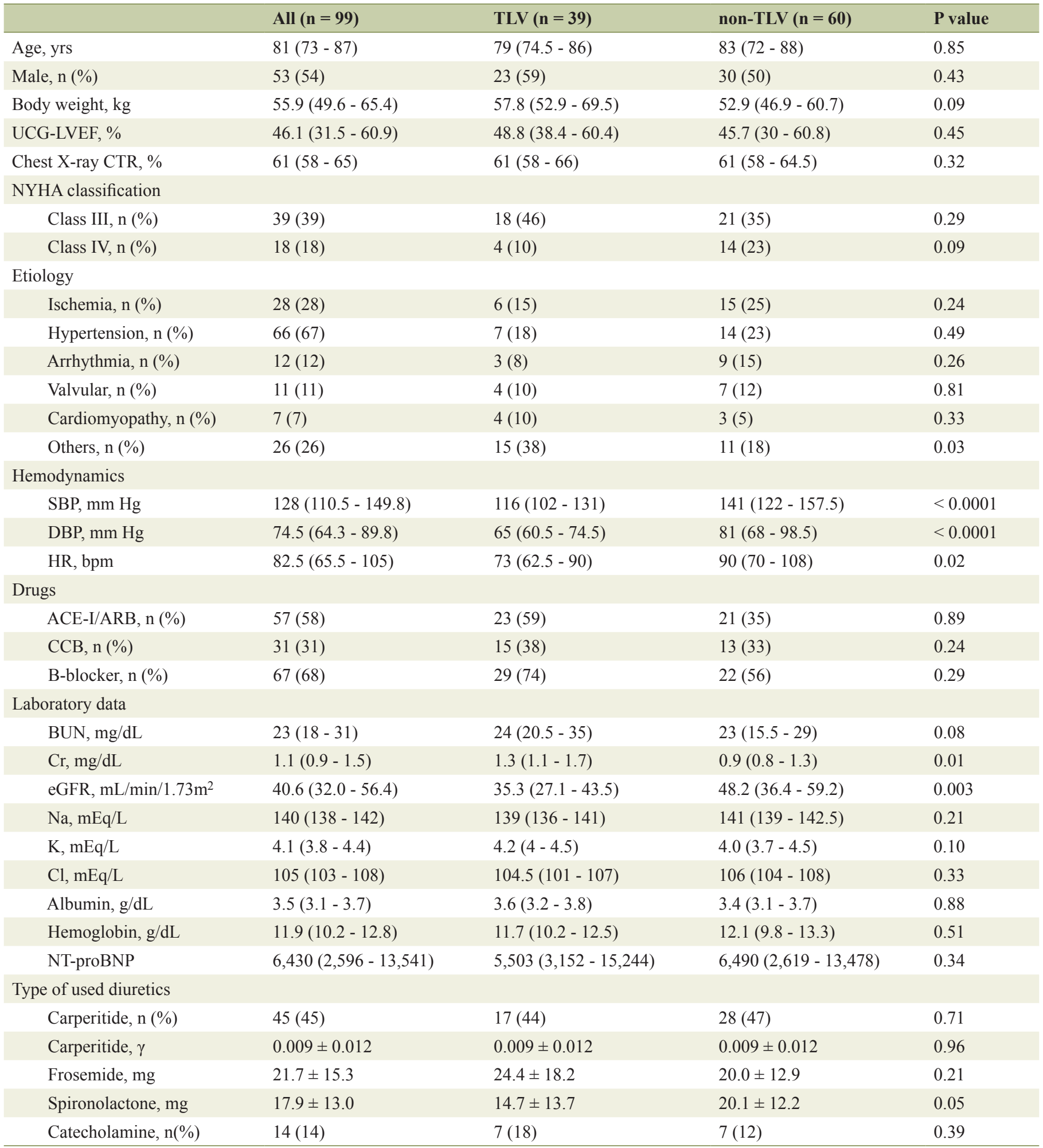

Data are shown as the mean \pm standard deviation (SD) or $\mathrm{n}(\%)$ or as the median (interquartile range). UCG-LVEF: ultrasonic cardiogram-left ventricular ejection fraction; CTR: cardiac thoracic ratio; NYHA: New York Heart Association; SBP: systolic blood pressure; DBP: diastolic blood pressure; HR: heart rate; ACE-I/ARB: angiotensin converting enzyme inhibitor/angiotensin receptor blocker; CCB: calcium channel blocker; BUN: blood urea nitrogen; Cr: creatinine; eGFR: estimated glomerular filtration rate; Na: sodium; K: potassium; Cl: chloride ion; NT-proBNP: n-terminal pro b-type natriuretic peptide. 

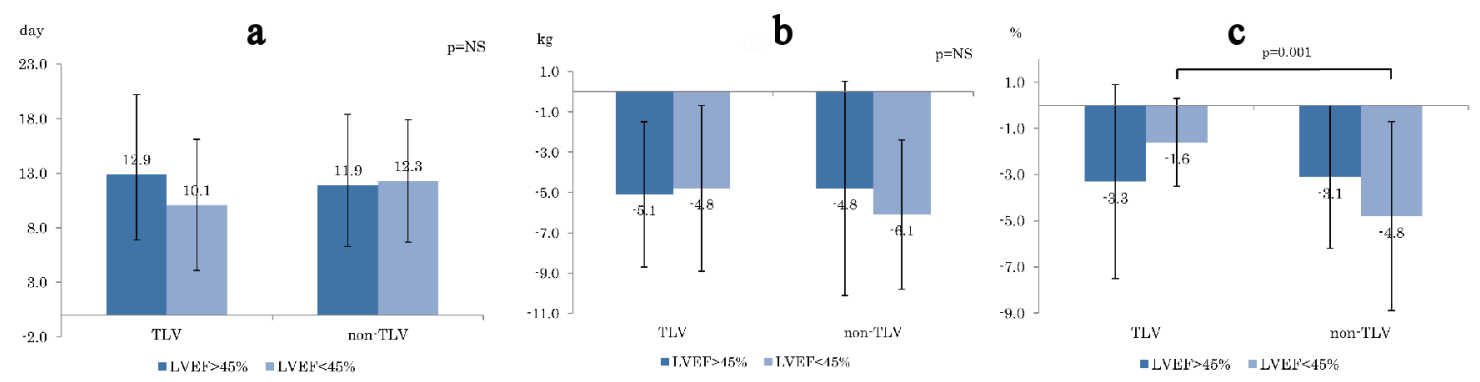

Figure 1. Comparison of (a) days to heart failure improvement, (b) BW reduction , and (c) CTR reducing between TLV and nonTLV. BW: body weight. CTR: cardiac thoracic ratio; LVEF: left ventricular ejection fraction.

\section{Changes in blood pressure 1 week before and after treat- ment}

Figure 2c shows changes in blood pressure (SBP (Fig. 2c-1) and DBP (Fig. 2c-2)) and HR (Fig. 2c-3) from pre-treatment to 1 week after the treatment. Both SBP and DBP were significantly higher in the non-TLV group until the second day, whereas HR was significantly higher in the non-TLV group only on the first day, and there was no significant difference in both BP and HR in the following day.

\section{Changes in renal function parameters and WRF rate prior to treatment and 1 week after treatment}

Figure 3a-c shows changes in renal function ((a): $\triangle B U N,(b)$ :
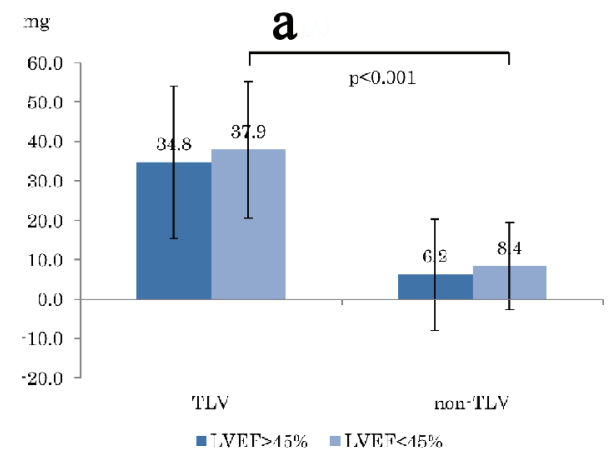

$\Delta \mathrm{Cr},(\mathrm{c}): \Delta \mathrm{eGFR})$. The $\Delta \mathrm{Cr}$ in the non-TLV group was significantly increased compared to that in the TLV group in terms of LVEF $>45 \%$ (TLV: $-0.1 \mathrm{mg} / \mathrm{dL}$, non-TLV: $+0.2 \mathrm{mg} / \mathrm{dL}, \mathrm{P}=$ 0.02 ), whereas $\triangle \mathrm{eGFR}$ in the non-TLV group was significantly decreased in terms of LVEF $>45 \%$ (TLV: $+1.4 \mathrm{~mL} / \mathrm{min} / 1.73$ $\mathrm{m}^{2}$, non-TLV: $\left.-2.7 \mathrm{~mL} / \mathrm{min} / 1.73 \mathrm{~m}^{2}, \mathrm{P}=0.04\right)$. There was no difference in the changes in serum electrolytes levels between the two groups $(\Delta \mathrm{Na}: \mathrm{P}=0.29, \Delta \mathrm{K}: \mathrm{P}=0.63)$.

Figure $3 \mathrm{~d}$ shows the WRF rate. The WRF rate in the TLV group was significantly lower than that in the non-TLV group in LVEF > 45\% (TLV: $2.5 \%$, non-TLV: $15.4 \%, \mathrm{P}=0.01$ ), whereas the WRF rate of the LVEF $<45 \%$ group showed similar tendency as in LVEF $>45 \%$ group, but it was not significant.

In Figure $3 b-d$, there were significant differences in all patient groups (TLV vs. non-TLV, P value, respectively; (3b): -0.1 $\mathrm{mg} / \mathrm{dL}$ vs. $+0.2 \mathrm{mg} / \mathrm{dL}, \mathrm{P}=0.03 ;(3 \mathrm{c}):+1.0 \mathrm{~mL} / \mathrm{min} / 1.73 \mathrm{~m}^{2} \mathrm{vs}$.

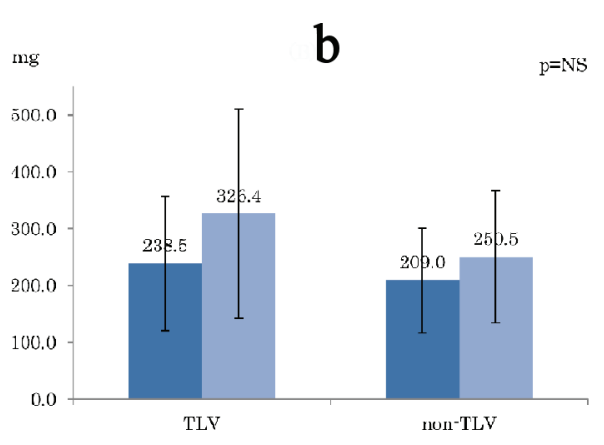

- $L V E F>45 \% \quad-\mathrm{LVEF}<45 \%$
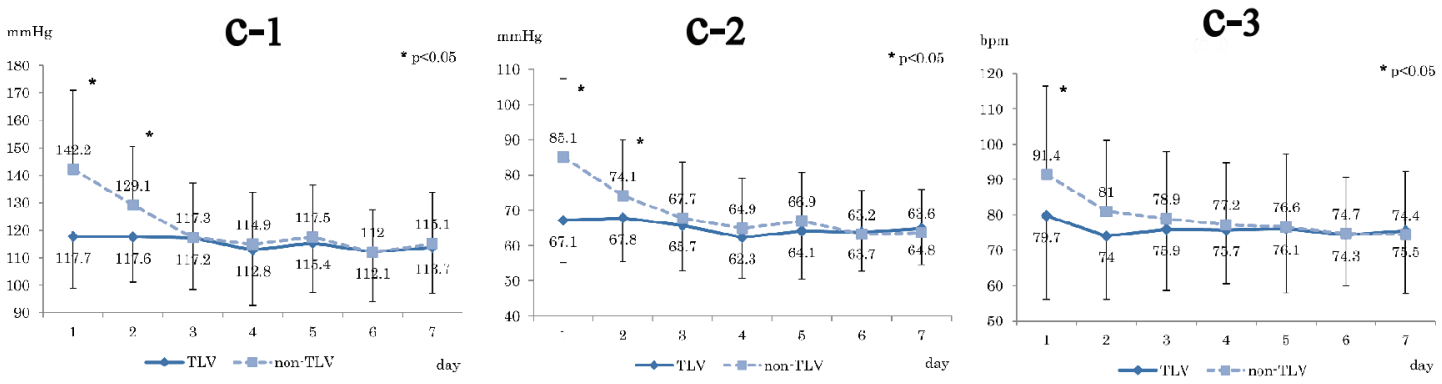

Figure 2. (a) Averaged loop diuretic dose before hospitalization. (b) Total loop diuretic used dose for 1 week from the start of treatment. (c) Blood pressure changes during 1 week compared TLV and non-TLV. (c-1) Systolic blood pressure. (c-2) Diastolic blood pressure. (c-3) Heart rate. Day: number of days since start TLV treatment; LVEF: left ventricular ejection fraction. 

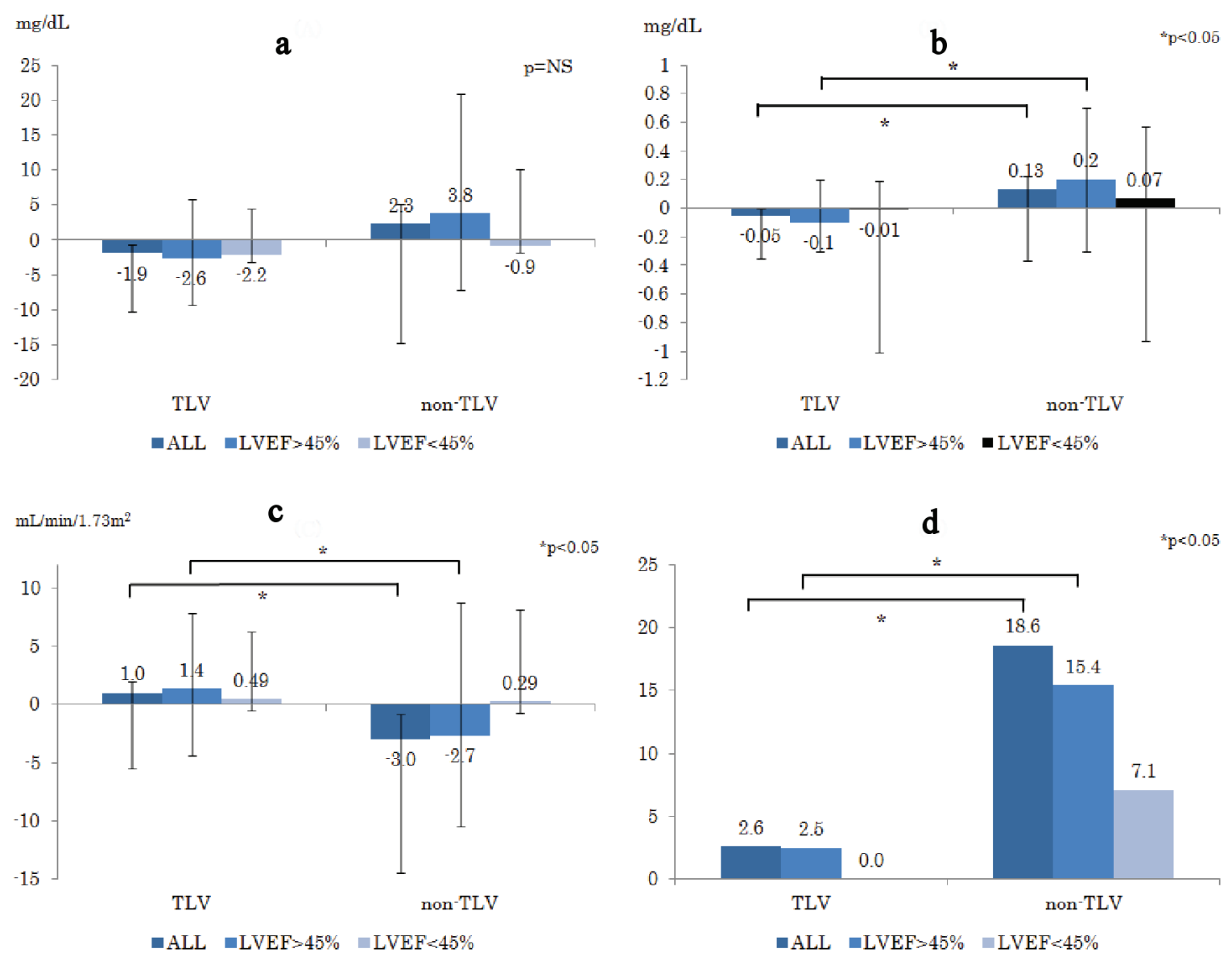

Figure 3. Changes in parameters of renal function prior to the treatment and 1 week after the treatment ((a): $\triangle B U N,(b): \Delta C r$, (c): $\triangle$ eGFR). (d) WRF appearance rate (\%) during hospitalization. WRF is defined as increase in serum creatinine levels at $0.3 \mathrm{mg} /$ $\mathrm{dL}$ and more. $\triangle \mathrm{BUN}$ : delta blood urea nitrogen; $\triangle \mathrm{Cr}$ : delta creatinine; $\triangle \mathrm{eGFR}$ : delta estimated glomerular filtration rate; WRF: worsening renal function.

$-2.7 \mathrm{~mL} / \mathrm{min} / 1.73 \mathrm{~m}^{2}, \mathrm{P}=0.04 ;(3 \mathrm{~d}): 2.6 \%$ vs. $18.6 \%, \mathrm{P}=$ 0.02 ), and the results of the LVEF $>45 \%$ group mainly contributed to the result of all patient comparison.

\section{Predictors of WRF by multiple logistic regression analysis}

We analyzed the associations between WRF and TLV administration by a logistic regression analysis (Table 2). As a result, among the candidate variables identified by univariate analysis, TLV was found to be an independent factor for reducing WRF (odds ratio: 0.14, 95\% CI: 0.02 - 0.98, $\mathrm{P}=0.04$ ).

\section{Discussion}

This study showed a significantly lower incidence of WRF despite higher doses of loop diuretics in the TLV group than that in the non-TLV group in AHF patients with CKD, suggesting the renoprotective effect of TLV. Moreover, this was a retrospective clinical study investigating TLV effectiveness in the real-world setting.
The mean eGFR at baseline was $43 \mathrm{~mL} / \mathrm{min} / 1.73 \mathrm{~m}^{2}$, and most patients exhibited moderate to severe renal insufficiency at the stage G3b of CKD classification. Patients in the TLV group displayed significantly lower eGFR compared to patients in the non-TLV group (Table 1), probably due to the higher average dose of loop diuretics used before hospitalization (Fig. 2a).

Therefore, the reason for the lower eGFR in the TLV group was that TLV was used for patients who showed loop diuretic treatment resistance by corresponding doctors. Hence, the TLV group needs to have TLV combined with loop diuretics, and it is considered that the TLV group had poor response to loop diuretics due to renal dysfunction. This finding sug-

Table 2. Independent Predictors of Worsening Renal Function

\begin{tabular}{llll} 
& Odds ratio & $\mathbf{9 5 \%}$ CI & P value \\
\hline HT & 2.07 & $0.49-8.72$ & 0.32 \\
eGFR & 1.36 & $0.26-7.25$ & 0.72 \\
Tolvaptan & 0.14 & $0.02-0.98$ & 0.04 \\
\hline
\end{tabular}

$\mathrm{Cl}$ : confidence interval; HT: hyper tension; eGFR: estimated glomerular filtration rate. 
gests that patients in the TLV group were resistant to loop diuretic treatment.

There was no significant difference in HF improvement period, body weight change, and CTR change except for CTR in LVEF $<45 \%$, suggesting a comparable improvement in HF in both groups (Fig. 1).

The higher total dose of loop diuretics from the beginning of HF treatment to 1 week after the treatment in TLV group (Fig. 2b) was due to the higher initial and before hospitalization dose of diuretics and the continuation of higher dose of loop diuretics in the TLV group (Table 1, TLV: $24.4 \mathrm{mg}$ vs. non-TLV: $20.0 \mathrm{mg}, \mathrm{P}=0.21$ ).

There was no significant differences in HF severity between the two groups based on NYHA classification at admission (Table 1, class III/IV TLV: $56 \%$ vs. non-TLV: $58 \%$, P = NS) and CTR at chest X-ray before treatment (Table 1, TLV: $61.0 \%$ vs. non-TLV: $61.0 \%, P=0.32$ ). In the non-TLV group, $\triangle \mathrm{BUN}$ and $\Delta \mathrm{Cr}$ were higher and $\Delta \mathrm{eGFR}$ was lower than those in the TLV group, indicating that the incidence of WRF was significantly higher than that in the TLV group. On the contrary, although the patients in the TLV group were resistant to loop diuretic treatment, the WRF rate was significantly lower than that in the non-TLV group at LVEF $>45 \%$ (Fig. 3d). In combination with TLV, an effective therapeutic effect without decreasing the dose of loop diuretics is demonstrated, and WRF can be possibly suppressed. This result was completely corresponding to the K-STAR study [11].

Regarding the hemodynamic effect of TLV treatment, the SBP, DBP, and HR were decreased in the non-TLV group, but they remained at the same levels in the TLV group throughout the 1-week treatment (Fig. 2c1-c3). This was because both SBP and DBP in the non-TLV group at hospitalization were significantly higher than those in the TLV group (Table 1), suggesting an increased treatment effect on HF. On the contrary, SBP and DPB in the TLV group at hospitalization were within the normal range, and both SBP and DBP remained at the same level, indicating that TLV did not affect hemodynamics and is a safe treatment option for HF.

The results in the present study suggest that TLV is an effective treatment option for loop diuretic-resistant AHF patients with comorbid CKD and has a renoprotective effect, in which WRF rate is reduced even under high-dose loop diuretics. In addition, TLV has less depressor effect, suggesting that it is a safe therapeutic agent for HF.

CKD is the most important risk of WRF $[13,14]$. WRF exacerbates prognosis even if it is temporary [15]. In a recent report, TLV reduces WRF in treatment of AHF with CKD [16]. TLV is effective in treating heart failure with CKD, continuous administration of TLV improves middle-term prognosis [17].

In this study, TLV was an independent contributing factor that decreases WRF based on multivariate analysis (Table 2). Furthermore, we examined HFrEF and HFpEF at a cutoff value of LVEF $45 \%$ and confirmed the renoprotective effect in HFpEF. TLV was likely to have a renoprotective effect in both HFrEF and HFpEF because it did not show renal dysfunction in HFrEF. The clinical treatment of HFrEF patient with CKD is very difficult and easily complicates WRF due to reduction of renal blood flow. Therefore, it is quite important that use of TLV does not cause impairment of renal function in HFrEF with CKD.

Long-term efficacy of TLV in HFpEF has recently been reported [18]. In this study, TLV suppresses acute WRF of HF$\mathrm{pEF}$, and suppression of acute WRF was a meaningful result because it was involved in long-term efficacy.

Although the present study is a retrospective study, our results provide critical information since TLV treatment in daily clinical setting is considered for diuretic-resistant patients with comorbid CKD who have poor response to loop diuretics. Our present data reconfirmed that TLV was important as an initial therapeutic strategy for loop diuretic-resistant patients with acute decompensated HF.

\section{Study limitations}

There were few study limitations. First, it is a retrospective study which does not have sufficient data of urine-related parameters (urine excretion amount of electrolytes, $\mathrm{Cr}$, osmotic pressure, free water clearance); thus, no systematic evaluation of TLV effect on renal function was carried out. Second, this was a single-site study, and the sample size was moderate (n =99), which may not have sufficient statistical power. Therefore, a prospective multi-center study of TLV effect in diureticresistant patients is required.

\section{Conclusions}

This study showed significantly lower incidence of WRF despite higher doses of loop diuretics in the TLV group compared to the non-TLV group in AHF patients with CKD, especially in HFpEF. Our results suggest that TLV is a valuable therapeutic agent for diuretic-resistant AHF patients with potential renoprotective effect.

\section{Funding}

This research received no grant from any funding agency in the public, commercial, or not-for-profit sectors.

\section{Disclosure}

All authors have no relationships relevant to the contents of this paper to disclose.

\section{Financial Support}

\section{Nothing}

\section{References}

1. Okura Y, Ramadan MM, Ohno Y, Mitsuma W, Tanaka K, Ito M, Suzuki K, et al. Impending epidemic: future pro- 
jection of heart failure in Japan to the year 2055. Circ J. 2008;72(3):489-491.

2. McMurray JJ, Adamopoulos S, Anker SD, Auricchio A, Bohm M, Dickstein K, Falk V, et al. ESC Guidelines for the diagnosis and treatment of acute and chronic heart failure 2012: The Task Force for the Diagnosis and Treatment of Acute and Chronic Heart Failure 2012 of the European Society of Cardiology. Developed in collaboration with the Heart Failure Association (HFA) of the ESC. Eur Heart J. 2012;33(14):1787-1847.

3. Sato N, Kajimoto K, Keida T, Mizuno M, Minami Y, Yumino D, Asai K, et al. Clinical features and outcome in hospitalized heart failure in Japan (from the ATTEND Registry). Circ J. 2013;77(4):944-951.

4. Felker GM, Lee KL, Bull DA, Redfield MM, Stevenson LW, Goldsmith SR, LeWinter MM, et al. Diuretic strategies in patients with acute decompensated heart failure. N Engl J Med. 2011;364(9):797-805.

5. O'Connor CM, Starling RC, Hernandez AF, Armstrong PW, Dickstein K, Hasselblad V, Heizer GM, et al. Effect of nesiritide in patients with acute decompensated heart failure. N Engl J Med. 2011;365(1):32-43.

6. Chen HH, Anstrom KJ, Givertz MM, Stevenson LW, Semigran MJ, Goldsmith SR, Bart BA, et al. Low-dose dopamine or low-dose nesiritide in acute heart failure with renal dysfunction: the ROSE acute heart failure randomized trial. JAMA. 2013;310(23):2533-2543.

7. Gheorghiade M, Konstam MA, Burnett JC, Jr., Grinfeld L, Maggioni AP, Swedberg K, Udelson JE, et al. Short-term clinical effects of tolvaptan, an oral vasopressin antagonist, in patients hospitalized for heart failure: the EVEREST Clinical Status Trials. JAMA. 2007;297(12):13321343.

8. Gottlieb SS, Stebbins A, Voors AA, Hasselblad V, Ezekowitz JA, Califf RM, O'Connor CM, et al. Effects of nesiritide and predictors of urine output in acute decompensated heart failure: results from ASCEND-HF (acute study of clinical effectiveness of nesiritide and decompensated heart failure). J Am Coll Cardiol. 2013;62(13):11771183.

9. Konstam MA, Gheorghiade M, Burnett JC, Jr., Grinfeld L, Maggioni AP, Swedberg K, Udelson JE, et al. Effects of oral tolvaptan in patients hospitalized for worsening heart failure: the EVEREST Outcome Trial. JAMA.
2007;297(12):1319-1331.

10. Matsue Y, Suzuki M, Torii S, Yamaguchi S, Fukamizu S, Ono Y, Fujii H, et al. Clinical Effectiveness of Tolvaptan in Patients With Acute Heart Failure and Renal Dysfunction. J Card Fail. 2016;22(6):423-432.

11. Inomata T, Ikeda Y, Kida K, Shibagaki Y, Sato N, Kumagai Y, Shinagawa H, et al. Effects of Additive Tolvaptan vs. Increased Furosemide on Heart Failure With Diuretic Resistance and Renal Impairment- Results From the KSTAR Study. Circ J. 2017;82(1):159-167.

12. Hanatani A, Shibata A, Kitada R, Iwata S, Matsumura Y, Doi A, Sugioka K, et al. Administration of tolvaptan with reduction of loop diuretics ameliorates congestion with improving renal dysfunction in patients with congestive heart failure and renal dysfunction. Heart Vessels. 2017;32(3):287-294.

13. Hsu CY, Ordonez JD, Chertow GM, Fan D, McCulloch $\mathrm{CE}, \mathrm{Go} A \mathrm{~S}$. The risk of acute renal failure in patients with chronic kidney disease. Kidney Int. 2008;74(1):101-107.

14. Fonarow GC, Stough WG, Abraham WT, Albert NM, Gheorghiade M, Greenberg BH, O'Connor CM, et al. Characteristics, treatments, and outcomes of patients with preserved systolic function hospitalized for heart failure: a report from the OPTIMIZE-HF Registry. J Am Coll Cardiol. 2007;50(8):768-777.

15. Uchino S, Bellomo R, Bagshaw SM, Goldsmith D. Transient azotaemia is associated with a high risk of death in hospitalized patients. Nephrol Dial Transplant. 2010;25(6):1833-1839.

16. Ikeda S, Ohshima K, Miyazaki S, Kadota H, Shimizu H, Ogimoto A, Hamada M. Impact of chronic kidney disease on the diuretic response of tolvaptan in acute decompensated heart failure. ESC Heart Fail. 2017;4(4):614-622.

17. Nakano Y, Mizuno T, Niwa T, Mukai K, Wakabayashi $\mathrm{H}$, Watanabe A, Ando H, et al. Impact of continuous administration of tolvaptan on preventing medium-term worsening renal function and long-term adverse events in heart failure patients with chronic kidney disease. Int Heart J. 2018;59(1):105-111.

18. Imamura $\mathrm{T}$, Kinugawa $\mathrm{K}$. Tolvaptan improves the longterm prognosis in patients with congestive heart failure with preserved ejection fraction as well as in those with reduced ejection fraction. Int Heart J. 2016;57(5):600606. 